\title{
Gap Invariance of a Symmetric Invariant Lamination
}

\author{
Masamichi YOSHIDA \\ Osaka City University \\ (Communicated by Y. Ito)
}

\begin{abstract}
We shall show that any symmetric, forward and backward invariant lamination under $z \mapsto z^{d}$ is gap invariant in the sense of W. P. Thurston. As a corollary, we have the gap invariance of a quadratic invariant lamination.
\end{abstract}

\section{Introduction.}

Let $\overline{\mathbf{D}}$ (resp. D) be the closed (resp. open) unit disk in the complex plane C. For each subset $A$ of $\overline{\mathbf{D}}$, denote the convex hull of $A$ by $\operatorname{co} A$, and let $-A=\{z \in \overline{\mathbf{D}} \mid-z \in A\}$. We call a subset $S$ of $\overline{\mathbf{D}}$ a chord if $S=c o\{\zeta, \eta\}$ for some $\zeta$ and $\eta$ in $\partial \mathbf{D}$ (we often write $S=\overline{\zeta \eta}$ if $\zeta \neq \eta$ ), and $S$ a degenerate chord if $\zeta=\eta$. When $S=\overline{\zeta \eta}$, let $\operatorname{ex} S=\{\zeta, \eta\}$. Following Thurston ([4] and [1]), a lamination $\mathcal{L}$ is a family of chords (an element of $\mathcal{L}$ is called a leaf) such that $\cup \mathcal{L}$ is closed in $\mathbf{C}$, and $\mathcal{L}$ is non-crossing i.e. no two distinct leaves intersect in $\mathbf{D}$. $\mathbf{A}$ gap of $\mathcal{L}$ is the closure of a component of $\overline{\mathbf{D}} \backslash(\cup \mathcal{L})$.

For each positive integer $d$ with $d \geq 2$, define a mapping $p_{d}: \partial \mathbf{D} \rightarrow \partial \mathbf{D}$ by $p_{d}(\zeta)=\zeta^{d}$. For a non-degenerate chord $S$, define $P_{d} S=\operatorname{co} p_{d}(\operatorname{ex} S)$. A lamination $\mathcal{L}$ is said to be forward invariant under $p_{d}$ if for any $S \in \mathcal{L}, P_{d} S \in \mathcal{L}$ or $P_{d} S$ is degenerate. Furthermore $\mathcal{L}$ is said to be backward invariant if for any $S=\overline{p q} \in \mathcal{L}$, there is a collection of $d$ disjoint chords in $\mathcal{L}$, each joining an inverse image of $p$ to an inverse image of $q$. Finally $\mathcal{L}$ is said to be gap invariant if $\operatorname{co~}_{p_{d}}(G \cap \partial \mathbf{D})$ is a gap of $\mathcal{L}$, a leaf, or degenerate for any gap $G$ of $\mathcal{L}$.

Consider the case $d=2$. Write $h=p_{2}$. In this case, for a forward invariant lamination $\mathcal{L}$, its backward invariance is equivalent to the following property: for each nondegenerate leaf $S,-S \in \mathcal{L}$ and there is $R \in \mathcal{L}$ such that $h R=S$. Indeed suppose $\mathcal{L}$ is backward invariant. It suffices to show that $-S \in \mathcal{L}$ for each non-degenerate $S \in \mathcal{L}$. When $S$ is a diameter, it is clear. Suppose $S$ is not a diameter. Since $h S$ is non-degenerate, $h S \in \mathcal{L}$ by the forward invariance. By the backward invariance, $-S \in \mathcal{L}$. The converse is clear.

C. Bandt and K. Keller constructed a forward and backward invariant lamination under $h$ and states, in Theorem 5.2 ([1]), that it is gap invariant. In this paper, we shall give the proof of the gap invariance theorem for symmetric and (forward and backward) invariant lamination 
under $p_{d}$ where $d \geq 2$ (see Section 3), and indicate some systematic approach to study gaps of a lamination. To end the introduction, we mention that a result of K. M. Pilgrim ([2, p. 1324]) states that there is a lamination which fails to be gap invariant under $h$.

\section{Boundary chords of a gap and 1-sided families.}

For each $x \in \mathbf{C}$ and $\varepsilon>0$, denote $B_{\varepsilon}(x)=\{y \in \mathbf{C}|| y-x \mid<\varepsilon\}$. For each subset $A$ of $\mathbf{C}$, denote by $c l A$ (resp. int $A$ ) the closure (resp. interior) of $A$ in $\mathbf{C}$ and for each $B \subset A$, denote by $\operatorname{int}_{A} B$ (resp. $\partial_{A} B$ ) the relative interior (resp. relative boundary) of $B$ in $A$ (notice that the relative closure of $B$ in $A$ is $A \cap c l B$ ). Since $\overline{\mathbf{D}}$ is closed in $\mathbf{C}$, we have $c l B=\operatorname{int}_{\overline{\mathbf{D}}} B \cup \partial_{\overline{\mathbf{D}}} B=$ $B \cup \partial_{\overline{\mathbf{D}}} B$ for each $B \subset \overline{\mathbf{D}}$. For each $B \subset \partial \mathbf{D}$, we write int $B=\operatorname{int}_{\partial \mathbf{D}} B$ and $\tilde{\partial} B=\partial_{\partial \mathbf{D}} B$. Similarly since $\partial \mathbf{D}$ is closed in $\mathbf{C}$, we have $c l B=\tilde{\text { int }} B \cup \tilde{\partial} B=B \cup \tilde{\partial} B$ for each $B \subset \partial \mathbf{D}$. Denote $\mathcal{S}=\{S \subset \overline{\mathbf{D}} \mid S$ is a chord $\}$ and $\mathcal{S}_{+}=\{S \in \mathcal{S} \mid S$ is non-degenerate $\}$. For $S \in \mathcal{S}_{+}$, denote $S^{\circ}=S \backslash e x S$, and for each connected subset $V$ of $\overline{\mathbf{D}} \backslash S$, the connected component of $\overline{\mathbf{D}} \backslash S$ containing $V$ by $D_{V}\langle S\rangle$, in particular when $V=\{x\}$, write $D_{x}\langle S\rangle=D_{V}\langle S\rangle$. We often use the following fact. Suppose that $l \subset \overline{\mathbf{D}}$ is a non-degenerate line segment (for example, $l=c o\{z, w\}$ for some $z, w \in \overline{\mathbf{D}}$ with $z \neq w)$. Let $A \subset \overline{\mathbf{D}}$. If $l \cap A \neq \emptyset$ and $l \backslash A \neq \emptyset$, then $l \cap \partial_{\overline{\mathbf{D}}} A \neq \emptyset$ (indeed notice that $l$ is connected).

We also use the following general lemmas (see Appendix). Let $X$ be a topological space.

LEMMA 1. Let $D$ be a connected and open subset of $X$ and $E$ is a non-empty subset of D. If $\partial E \subset \partial D$, then $E=D$.

LEMMA 2. Let $E \subset X$. Then $\partial E \supset \partial \mathrm{cl} E$. Furthermore $\partial E \subset \partial c l E$ if and only if $E \supset$ int $c l E$.

For each $E \subset X$, denote by $\operatorname{Comp}(E)$ the family of connected components of $E . X$ is said to be locally connected if for any open subset $U$ of $X$ and any $C \in \operatorname{Comp}(U), C$ is open in $X$. Hence $\overline{\mathbf{D}}$ and $\partial \mathbf{D}$ is locally connected with respect to their relative topology.

LEMMA 3. Suppose that $X$ is locally connected. Let $F$ be a closed subset of $X$. Then for any $C \in \operatorname{Comp}(X \backslash F), \partial C \subset F$. Furthermore $\partial F=\operatorname{cl}\left(\bigcup_{C \in \operatorname{Comp}(X \backslash F)} \partial C\right)$.

For a subfamily $\mathcal{M}$ of $\mathcal{S}$, define $\mathcal{M}_{+}=\mathcal{M} \cap \mathcal{S}_{+}, \mathcal{M}^{\circ}=\left\{S^{\circ} \mid S \in \mathcal{M}_{+}\right\}$and ex $\mathcal{M}=$ $\partial \mathbf{D} \cap\left(\cup \mathcal{M}_{+}\right)$. Then $(\cup \mathcal{M}) \cap \mathbf{D}=\cup \mathcal{M}^{\circ}$ and $\operatorname{ex} \mathcal{M}=\left\{x \in \partial \mathbf{D} \mid x \in S\right.$ for some $\left.S \in \mathcal{M}_{+}\right\}$.

DEFINITION 1 (Non-crossing family of chords and Lamination). Suppose a subfamily $\mathcal{L}$ of $\mathcal{S}$ satisfies that $\mathcal{L}_{+} \neq \emptyset$ and $\overline{\mathbf{D}} \backslash \operatorname{cl}(\cup \mathcal{L}) \neq \emptyset$ (hence $\mathbf{D} \backslash \cup \mathcal{L} \neq \emptyset$ ). We say $\mathcal{L}$ is noncrossing if for any $R, S \in \mathcal{L}_{+}, R=S$ or $S^{\circ} \cap R=\emptyset$, or equivalently if for any (or some) $x \in \mathbf{D} \backslash \cup \mathcal{L}$ and any $R \neq S \in \mathcal{L}_{+}, S^{\circ} \subset D_{x}\langle R\rangle$ or $S^{\circ} \cap$ cl $D_{x}\langle R\rangle=\emptyset$. Let $\mathcal{L}$ be non-crossing. For each $V \in \operatorname{Comp}(\overline{\mathbf{D}} \backslash \cup \mathcal{L})$, define $\mathcal{L}_{V}=\left\{S \in \mathcal{S}_{+} \mid S \subset \partial_{\overline{\mathbf{D}}} V\right\}$. We say $\mathcal{L}$ is a lamination if $\mathcal{L}$ is non-crossing and $\cup \mathcal{L}$ is closed in $\mathbf{C}$.

REMARK 1. Let $\mathcal{L}$ be a lamination and $V \in \operatorname{Comp}(\overline{\mathbf{D}} \backslash \cup \mathcal{L})$. Then $V$ is relatively open in $\overline{\mathbf{D}}, \partial_{\overline{\mathbf{D}}} V \subset \cup \mathcal{L}, \mathbf{D} \cap \partial_{\overline{\mathbf{D}}} V=\cup \mathcal{L}_{V}^{\circ}$, and furthermore $\sharp \mathcal{L}_{V} \geq 1$. 
Proof. By Lemma 3, $V$ is relatively open in $\overline{\mathbf{D}}$ and $\partial_{\overline{\mathbf{D}}} V \subset \cup \mathcal{L}$ because $\overline{\mathbf{D}}$ is locally connected and $\cup \mathcal{L}$ is relatively closed in $\overline{\mathbf{D}}$. Hence $\mathbf{D} \cap \partial_{\overline{\mathbf{D}}} V=\cup \mathcal{L}_{V}^{\circ}$. (Indeed $\cup \mathcal{L}_{V}^{\circ}=$ $\mathbf{D} \cap\left(\cup \mathcal{L}_{V}\right) \subset \mathbf{D} \cap \partial_{\overline{\mathbf{D}}} V$. Conversely let $x \in \mathbf{D} \cap \partial_{\overline{\mathbf{D}}} V$. Since $x \in \mathbf{D} \cap(\cup \mathcal{L})$, there is $S \in \mathcal{L}$ such that $x \in S^{\circ}$. Assume $S \notin \mathcal{L}_{V}$. Let $y \in S \backslash \partial_{\overline{\mathbf{D}}} V$. So $y \notin V \cup \partial_{\overline{\mathbf{D}}} V=c l V$. Pick a point $z \in V$ (maybe $z \in \partial \mathbf{D})$. Then $l=(c o\{y, z\}) \backslash\{y, z\} \subset \mathbf{D} \cap D_{z}\langle S\rangle$. Since $y \notin c l V$, $l \backslash V \neq \emptyset$. Since $V$ is relatively open in $\overline{\mathbf{D}}, l \cap V \neq \emptyset$. Hence there is $w \in l \cap \partial_{\overline{\mathbf{D}}} V$. Since $w \in \mathbf{D} \cap(\cup \mathcal{L})$, there is $R \in \mathcal{L}$ such that $w \in R^{\circ}$. Since $w \in D_{z}\langle S\rangle$ and $\mathcal{L}$ is non-crossing, we have $S^{\circ} \cap \operatorname{cl} V \subset S^{\circ} \cap \operatorname{cl} D_{z}\langle R\rangle=\emptyset$, contradicting the fact that $x \in S^{\circ} \cap c l V$.)

Notice that $V \cap \mathbf{D}$ is relatively open, that is, $\operatorname{int}_{\overline{\mathbf{D}}}(V \cap \mathbf{D})=V \cap \mathbf{D}$. So $\partial_{\overline{\mathbf{D}}}(V \cap \mathbf{D})=$ $c l(V \cap \mathbf{D}) \backslash(V \cap \mathbf{D}) \subset(c l V \backslash V) \cup(c l V \backslash \mathbf{D}) \subset \partial_{\overline{\mathbf{D}}} V \cup \partial \mathbf{D}$. Assume $\mathcal{L}_{V}=\emptyset$. Then $\partial_{\overline{\mathbf{D}}} V \subset \partial \mathbf{D}$, hence $\partial_{\overline{\mathbf{D}}}(V \cap \mathbf{D}) \subset \partial \mathbf{D}=\partial_{\overline{\mathbf{D}}} \mathbf{D}$. By Lemma 1 , we have $V \cap \mathbf{D}=\mathbf{D}$, contradicting the assumption $\mathcal{L}_{+} \neq \emptyset$.

Let $\mathcal{L}$ be a lamination. A subset $G$ of $\overline{\mathbf{D}}$ is called a gap of $\mathcal{L}$ if $G=\operatorname{cl} V$ for some $V \in \operatorname{Comp}(\overline{\mathbf{D}} \backslash \cup \mathcal{L})$. We shall consider a representation for $G=c l V$ via a family $\mathcal{L}_{V}$. At first notice that $\mathcal{L}_{V} \subset \mathcal{L}_{+}$and since $V$ is relatively open in $\overline{\mathbf{D}}$,

$$
V \subset \operatorname{int}_{\overline{\mathbf{D}}}\left(\bigcap_{S \in \mathcal{L}_{V}} D_{V}\langle S\rangle\right) .
$$

Furthermore if $\sharp \mathcal{L}_{V} \geq 2$, then $\mathcal{L}_{V}$ is 1 -sided in the following sense.

DEFINITION 2 (1-sided family of chords). Let $\mathcal{N}$ be a non-crossing subfamily of $\mathcal{S}_{+}$ with $\sharp \mathcal{N} \geq 2$. We say that $\mathcal{N}$ is 1 -sided if $\cup \mathcal{N} \subset \operatorname{cl} D_{R^{\circ}}\langle S\rangle$ for any two distinct chords $R$ and $S$ in $\mathcal{N}$.

In order to get representation for gaps of a lamination, we shall study the property of a 1-sided family. Let $\mathcal{N}$ be 1-sided. Then for each $S \in \mathcal{N}$, the set $D_{R^{\circ}}\langle S\rangle$ is independent of the choice of $R \in \mathcal{N} \backslash\{S\}$ and so denote it by $D_{\mathcal{N}}(S)$. Furthermore the cardinality of $\mathcal{N}$ is at most countable. Indeed the first statement holds by the definition of 1 -sidedness. For each $S, R \in \mathcal{N}$ with $S \neq R$, we have that $\left(\overline{\mathbf{D}} \backslash c l D_{\mathcal{N}}(S)\right) \cap\left(\overline{\mathbf{D}} \backslash c l D_{\mathcal{N}}(R)\right)=\emptyset$. For each $S \in \mathcal{N}$, $\overline{\mathbf{D}} \backslash \operatorname{cl} D_{\mathcal{N}}(S)$ is relatively open in $\overline{\mathbf{D}}$. Since $\overline{\mathbf{D}}$ is separable, the second statement holds. We define the center of a 1 -sided family $\mathcal{N}$.

DEFINITION 3 (Center of a 1-sided family). Let $\mathcal{N}$ be a 1-sided subfamily of $\mathcal{S}_{+}$. Define $C_{\mathcal{N}}=\operatorname{int}_{\overline{\mathbf{D}}}\left(\bigcap_{S \in \mathcal{N}} D_{\mathcal{N}}(S)\right)$.

If $\mathcal{L}$ is a lamination, then $V \subset C_{\mathcal{L}_{V}}$ for each $V \in \operatorname{Comp}(\overline{\mathbf{D}} \backslash \cup \mathcal{L})$ with $\sharp \mathcal{L}_{V} \geq 2$, and $D_{\mathcal{L}_{V}}(S)=D_{V}\langle S\rangle$ for any $S \in \mathcal{L}_{V}$. At the end of this section, we shall show that $G=\operatorname{cl} V=$ $\operatorname{cl} C_{\mathcal{L}_{V}}$ (Lemma 5) and that if $\partial \mathbf{D}=\operatorname{clex} \mathcal{L}$, then $\sharp \mathcal{L}_{V} \geq 2$ for each $V \in \operatorname{Comp}(\overline{\mathbf{D}} \backslash \cup \mathcal{L})$ (Corollary 1).

REMARK 2. Let $\mathcal{N}$ be 1-sided. Then $C_{\mathcal{N}} \neq \emptyset$ and $\operatorname{cl}(\cup \mathcal{N}) \subset \operatorname{cl} C_{\mathcal{N}} \subset$ $\bigcap_{S \in \mathcal{N}} c l D_{\mathcal{N}}(S)$. 
PROOF. Let $S_{1}, S_{2}$ be distinct chords in $\mathcal{N}$. Since $\mathcal{N}$ is 1-sided, (int $\operatorname{co}\left(S_{1} \cup S_{2}\right)$ ) $\cap$ $(\cup \mathcal{N})=\emptyset$. Furthermore int $\operatorname{co}\left(S_{1} \cup S_{2}\right) \subset \bigcap_{S \in \mathcal{N}} D_{\mathcal{N}}(S)$. Hence $\emptyset \neq \operatorname{int} \operatorname{co}\left(S_{1} \cup S_{2}\right) \subset C_{\mathcal{N}}$ and $S_{1} \cup S_{2} \subset \mathrm{cl} C_{\mathcal{N}}$.

Denote $\mathcal{F}=\{F \mid F$ is a closed disconnected subset of $\partial \mathbf{D}$ with $\sharp F \geq 3\}$ and $\Gamma=\{\mathcal{N} \mid \mathcal{N}$ is a 1 -sided family\}. We can construct a 1 to 1 correspondence between $\Gamma$ and $\mathcal{F}$. Indeed if $F \in \mathcal{F}$, then the family

$$
\mathcal{N}_{F}=\{\operatorname{co} \tilde{\partial} \gamma \mid \gamma \in \operatorname{Comp}(\partial \mathbf{D} \backslash F)\},
$$

is 1 -sided (notice that $\gamma$ is an open arc for each $\gamma \in \operatorname{Comp}(\partial \mathbf{D} \backslash F)$ ). Conversely suppose $\mathcal{N}$ is 1-sided. Define

$$
F_{\mathcal{N}}=\bigcap_{S \in \mathcal{N}}\left(\partial \mathbf{D} \cap \operatorname{cl} D_{\mathcal{N}}(S)\right) .
$$

Then $\operatorname{Comp}\left(\partial \mathbf{D} \backslash F_{\mathcal{N}}\right)=\left\{\partial \mathbf{D} \backslash \operatorname{cl} D_{\mathcal{N}}(S)\right\}_{S \in \mathcal{N}}$ because $\left(\overline{\mathbf{D}} \backslash \operatorname{cl} D_{\mathcal{N}}(S)\right) \cap\left(\overline{\mathbf{D}} \backslash c l D_{\mathcal{N}}(R)\right)$ $=\emptyset$ for each $S, R \in \mathcal{N}$ with $S \neq R$. So $F_{\mathcal{N}}$ is closed disconnected with $\sharp F_{\mathcal{N}} \geq 3$. Since

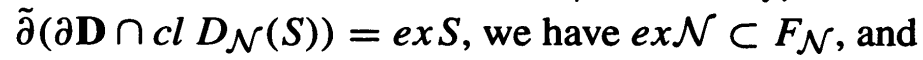

$$
\text { if } F=F_{\mathcal{N}} \text {, then } \mathcal{N}=\mathcal{N}_{F} \text {. }
$$

Notice that $\tilde{\partial} F_{\mathcal{N}}=c l e x \mathcal{N}$ by the local connectivity of $\partial \mathbf{D}$ and Lemma 3. Conversely let $F \in \mathcal{F}$. We have that

$$
\text { if } \mathcal{N}=\mathcal{N}_{F} \text {, then } F=F_{\mathcal{N}}
$$

because $\partial \mathbf{D} \backslash F=\bigcup_{S \in \mathcal{N}} \partial \mathbf{D} \backslash c l D_{\mathcal{N}}(S)=\cup \operatorname{Comp}\left(\partial \mathbf{D} \backslash F_{\mathcal{N}}\right)$. Thus we have a 1 to 1 correspondence $\mathcal{N} \leftrightarrow F_{\mathcal{N}}$. We shall show that if $\partial \mathbf{D}=\operatorname{cl}$ ex $\mathcal{L}$, then $\mathcal{L}_{V}=\mathcal{N}_{\partial \mathbf{D} \cap c l} v$ for each $V \in \operatorname{Comp}(\overline{\mathbf{D}} \backslash \cup \mathcal{L})$ (Corollary 1$)$. So we can reconstruct the family $\mathcal{L}_{V}$ from $\partial \mathbf{D} \cap$ cl $V$ by the above method. This equality $\mathcal{L}_{V}=\mathcal{N}_{\partial \mathrm{D} \cap c l} V$ is the key to prove gap invariance theorem (Section 3).

We use the following simple fact. Let $\varepsilon>0, x \in \partial \mathbf{D}$ and $S \in \mathcal{S}_{+}$. If $S \cap \operatorname{co}(\partial \mathbf{D} \cap$ $\left.B_{\varepsilon}(x)\right) \neq \emptyset$, then $\operatorname{ex} S \cap\left(\partial \mathbf{D} \cap B_{\varepsilon}(x)\right) \neq \emptyset$ (indeed if ex $S \subset \partial \mathbf{D} \backslash B_{\varepsilon}(x)$, then $S=\cos$ ex $\subset$ $\left.\operatorname{co}\left(\partial \mathbf{D} \backslash B_{\varepsilon}(x)\right) \subset \overline{\mathbf{D}} \backslash \operatorname{co}\left(\partial \mathbf{D} \cap B_{\varepsilon}(x)\right)\right)$. We also use Caratheodory's theorem (Theorem 17.1 in [3]): For any $A \subset \mathbf{C}$ and any $x \in \operatorname{co} A, x \in \operatorname{co}\left\{x_{1}, x_{2}, x_{3}\right\}$ for some $x_{1}, x_{2} . x_{3} \in A$. We show that $F_{\mathcal{N}}=\partial \mathbf{D} \cap c l C_{\mathcal{N}}, \widetilde{i n t} F_{\mathcal{N}}=\partial \mathbf{D} \cap C_{\mathcal{N}}, \tilde{\partial} F_{\mathcal{N}}=\partial \mathbf{D} \cap \partial_{\overline{\mathbf{D}}} C_{\mathcal{N}}$ and $c l C_{\mathcal{N}}=c o F_{\mathcal{N}}$. More precisely,

LEMMA 4. Let $\mathcal{N}$ be 1-sided. Then
a) $F_{\mathcal{N}}=\partial \mathbf{D} \cap c l C_{\mathcal{N}}$.
b) $\widetilde{\text { int }} F_{\mathcal{N}}=\partial \mathrm{D} \cap C_{\mathcal{N}}$.
c) $c l C_{\mathcal{N}}=c o F_{\mathcal{N}}$.
d) $\partial_{\overline{\mathbf{D}}} C_{\mathcal{N}}=\tilde{\partial} F_{\mathcal{N}} \cup\left(\cup \mathcal{N}^{\circ}\right)$.
e) $C_{\mathcal{N}}=\operatorname{int}_{\overline{\mathbf{D}}} c l C_{\mathcal{N}}$ (or equivalently, $\left.\partial_{\overline{\mathbf{D}}} C_{\mathcal{N}}=\partial_{\overline{\mathbf{D}}} c l C_{\mathcal{N}}\right)$.
f) $C_{\mathcal{N}} \in \operatorname{Comp}(\overline{\mathbf{D}} \backslash \operatorname{cl}(\cup \mathcal{N}))$.

Proof. Firstly we show the statements a) and b). By Remark 2, $F_{\mathcal{N}} \supset \partial \mathrm{D} \cap \mathrm{cl} C_{\mathcal{N}}$. Since $\cup \mathcal{N} \subset \operatorname{cl} C_{\mathcal{N}}$ (by Remark 2), we have $\tilde{\partial} F_{\mathcal{N}}=\operatorname{cl} \operatorname{ex} \mathcal{N} \subset \partial \mathbf{D} \cap \operatorname{cl} C_{\mathcal{N}}$. We show 
$\widetilde{\text { int }} F_{\mathcal{N}}=\partial \mathbf{D} \cap C_{\mathcal{N}}\left(\right.$ then $\left.F_{\mathcal{N}}=\partial \mathbf{D} \cap c l C_{\mathcal{N}}\right)$. If $x \in \partial \mathbf{D} \cap C_{\mathcal{N}}$, then $B_{\varepsilon_{0}}(x) \cap \partial \mathbf{D} \subset$ $\left(\bigcap_{s \in \mathcal{N}} D_{\mathcal{N}}(S)\right) \cap \partial \mathbf{D} \subset F_{\mathcal{N}}$ for some $\varepsilon_{0}>0$, hence $x \in \widetilde{\text { int }} F_{\mathcal{N}}$. Conversely let $x \in$ int $F_{\mathcal{N}}$. Then $\partial \mathbf{D} \cap B_{\varepsilon}(x) \subset \partial \mathbf{D} \backslash e x \mathcal{N}$ for some $\varepsilon>0$. Assume there is $S \in \mathcal{N}$ with $S \cap \operatorname{co}\left(\partial \mathbf{D} \cap B_{\varepsilon}(x)\right) \neq \emptyset$. Then ex $S \cap\left(\partial \mathbf{D} \cap B_{\varepsilon}(x)\right) \neq \emptyset$ contradicting the choice of $\varepsilon$. Hence $\operatorname{co}\left(\partial \mathbf{D} \cap B_{\varepsilon}(x)\right) \subset \overline{\mathbf{D}} \backslash \cup \mathcal{N}$. Since $x \in F_{\mathcal{N}} \subset \bigcap_{S \in \mathcal{N}} c l D_{\mathcal{N}}(S)$ and $\operatorname{co}\left(\partial \mathbf{D} \cap B_{\varepsilon}(x)\right)$ is connected and relatively open in $\overline{\mathbf{D}}$, we have $\operatorname{co}\left(\partial \mathbf{D} \cap B_{\varepsilon}(x)\right) \subset C_{\mathcal{N}}$.

c) We show the equality

$$
\bigcap_{S \in \mathcal{N}} \operatorname{cl} D_{\mathcal{N}}(S)=\operatorname{co} F_{\mathcal{N}}=\operatorname{cl} C_{\mathcal{N}}
$$

By Remark 2, it suffices to show $\bigcap_{s \in \mathcal{N}} \operatorname{cl} D_{\mathcal{N}}(S) \subset \operatorname{co} F_{\mathcal{N}} \subset \operatorname{cl} C_{\mathcal{N}}$. Let $x \in$ $\bigcap_{S \in \mathcal{N}} c l D_{\mathcal{N}}(S)$. If $x \in \partial \mathbf{D}$, then $x \in F_{\mathcal{N}}$. If $x \in \cup \mathcal{N}$, then $x \in S=\operatorname{coex} S$ for some $S \in \mathcal{N}$, hence $x \in \operatorname{co} F_{\mathcal{N}}$ since $\operatorname{ex} \mathcal{N} \subset F_{\mathcal{N}}$. Suppose $x \in \mathbf{D} \backslash \cup \mathcal{N}$. Let $\overline{a b} \in \mathcal{N}$ (note $\left.\{a, b\} \subset F_{\mathcal{N}}\right), \overline{a a^{\prime}}$ be the chord passing through $x, \overline{b b^{\prime}}$ the chord passing through $x$ and $\left[a^{\prime}, b^{\prime}\right]$ the closed arc between $a^{\prime}$ and $b^{\prime}$ containing neither $a$ nor $b$. If $\left[a^{\prime}, b^{\prime}\right] \cap F_{\mathcal{N}} \neq \emptyset$, then $x \in \Delta a b y \subset \operatorname{co} F_{\mathcal{N}}$ where $y \in\left[a^{\prime}, b^{\prime}\right] \cap F_{\mathcal{N}}$. If $\left[a^{\prime}, b^{\prime}\right] \subset \partial \mathbf{D} \backslash F_{\mathcal{N}}$, then $\left[a^{\prime}, b^{\prime}\right] \subset \gamma$ for some $\gamma \in \operatorname{Comp}\left(\partial \mathbf{D} \backslash F_{\mathcal{N}}\right)$. Note that $\gamma$ is an open arc and $\tilde{\partial} \gamma=\left\{a_{0}, b_{0}\right\} \subset F_{\mathcal{N}}$. Since $x \in \bigcap_{S \in \mathcal{N}} c l D_{\mathcal{N}}(S)$ and $\gamma \subset \partial \mathbf{D} \backslash F_{\mathcal{N}}$, we have that the points $a, a_{0}, b_{0}, b$ are distinct and $x \in \operatorname{co}\left\{a, a_{0}, b_{0}, b\right\} \subset \operatorname{co} F_{\mathcal{N}}$. Next we show $\operatorname{co} F_{\mathcal{N}} \subset c l C_{\mathcal{N}}$. It suffices, by the theorem of Caratheodory, to show for any (closed) triangle $\Delta$ whose vertices belong to $F_{\mathcal{N}}$, int $\Delta \subset C_{\mathcal{N}}$. Let $\Delta$ be such a triangle and $S \in \mathcal{N}$. If int $\Delta \backslash D_{\mathcal{N}}(S) \neq \emptyset$, then some vertex of $\Delta$ does not belong to $\partial \mathbf{D} \cap c l D_{\mathcal{N}}(S)\left(\supset F_{\mathcal{N}}\right)$ contradicting the choice of $\Delta$. Thus int $\Delta \subset \bigcap_{S \in \mathcal{N}} D_{\mathcal{N}}(S)$.

d) At first we show the equality

$$
\tilde{\partial} F_{\mathcal{N}}=\partial \mathbf{D} \cap \operatorname{cl}(\cup \mathcal{N}) \text {. }
$$

Since $\tilde{\partial} F_{\mathcal{N}}=c l e x \mathcal{N}, \tilde{\partial} F_{\mathcal{N}} \subset \partial \mathbf{D} \cap \operatorname{cl}(\cup \mathcal{N})$. Conversely let $x \in \partial \mathbf{D} \cap \operatorname{cl}(\cup \mathcal{N})$. Since $\operatorname{co}\left(\partial \mathbf{D} \cap B_{\varepsilon}(x)\right)$ is relatively open in $\overline{\mathbf{D}}$, we see that for any $\varepsilon>0, S \cap \operatorname{co}\left(\partial \mathbf{D} \cap B_{\varepsilon}(x)\right) \neq \emptyset$ for some $S \in \mathcal{N}$. So $B_{\varepsilon}(x) \cap e x S \neq \emptyset$, that is, $x \in \operatorname{cl} \operatorname{ex} \mathcal{N}=\tilde{\partial} F_{\mathcal{N}}$. We show the equality

$$
\tilde{\partial} F_{\mathcal{N}} \cup\left(\cup \mathcal{N}^{\circ}\right)=\partial_{\overline{\mathbf{D}}} C_{\mathcal{N}}=\operatorname{cl}(\cup \mathcal{N})
$$

By a) and Remark 2, $F_{\mathcal{N}} \cup \operatorname{cl}(\cup \mathcal{N}) \subset \operatorname{cl} C_{\mathcal{N}}$. It suffices to show $c l C_{\mathcal{N}} \backslash\left(\tilde{\partial} F_{\mathcal{N}} \cup\left(\cup \mathcal{N}^{\circ}\right)\right)=$ $C_{\mathcal{N}}=\operatorname{cl} C_{\mathcal{N}} \backslash \operatorname{cl}(\cup \mathcal{N})$. We show that $x \in C_{\mathcal{N}}$ for each $x \in \operatorname{cl} C_{\mathcal{N}} \backslash\left(\tilde{\partial} F_{\mathcal{N}} \cup\left(\cup \dot{\mathcal{N}}^{\circ}\right)\right)$. If $x \in \partial \mathbf{D}$, then $x \in \widetilde{\text { int }} F_{\mathcal{N}} \subset C_{\mathcal{N}}$ by a). Suppose $x \in \mathbf{D}$. By (1), $x \in \bigcap_{S \in \mathcal{N}} c l D_{\mathcal{N}}(S)$. Assume that $B_{\varepsilon}(x) \cap(\cup \mathcal{N}) \neq \emptyset$ for any $\varepsilon>0$ with $B_{\varepsilon}(x) \subset$ D. Then since $x \notin \cup \mathcal{N}^{\circ}$, $\sharp\left\{S \in \mathcal{N} \mid B_{\varepsilon}(x) \cap S \neq \emptyset\right\}=\infty$. In particular $B_{\varepsilon}(x) \cap S_{i} \neq \emptyset$ for some $S_{1}, S_{2}, S_{3} \in \mathcal{N}$ with $S_{i}^{\circ} \cap S_{j}^{\circ}=\emptyset(i \neq j)$. We can suppose $S_{3} \subset\left(c l D_{S_{2}^{\circ}}\left\langle S_{1}\right\rangle\right) \cap\left(c l D_{S_{1}^{\circ}}\left\langle S_{2}\right\rangle\right)$. Hence $D_{S_{1}^{\circ}}\left\langle S_{3}\right\rangle \cap D_{S_{2}^{\circ}}\left\langle S_{3}\right\rangle=\emptyset$, contradicting the 1-sidedness of $\mathcal{N}$. So $B_{\varepsilon_{0}}(x) \subset \mathbf{D} \backslash \cup \mathcal{N}$ for some $\varepsilon_{0}>0$. Since $B_{\varepsilon_{0}}(x)$ is connected and $x \in \bigcap_{S \in \mathcal{N}} c l D_{\mathcal{N}}(S)$, we have $B_{\varepsilon_{0}}(x) \subset$ $\bigcap_{S \in \mathcal{N}} D_{\mathcal{N}}(S)$, in particular $x \in C_{\mathcal{N}}$.

Next we show $C_{\mathcal{N}} \subset \operatorname{cl} C_{\mathcal{N}} \backslash \operatorname{cl}(\cup \mathcal{N})$, that is, $C_{\mathcal{N}} \cap \operatorname{cl}(\cup \mathcal{N})=\emptyset$. If $x \in C_{\mathcal{N}} \cap \partial \mathbf{D}$, then $x \in \widetilde{\text { int }} F_{N}$ by b). Since $x \in \partial \mathbf{D}, x \notin \operatorname{cl}(\cup \mathcal{N})$ by (2). When $x \in C_{\mathcal{N}} \cap \mathbf{D}$ we have $B_{\varepsilon_{0}}(x) \subset \bigcap_{S \in \mathcal{N}} D_{\mathcal{N}}(S)$ for some $\varepsilon_{0}>0$, so $B_{\varepsilon_{0}}(x) \cap(\cup \mathcal{N})=\emptyset$ i.e. $x \notin \operatorname{cl}(\cup \mathcal{N})$. 
Finally by (2), we have $c l C_{\mathcal{N}} \backslash \operatorname{cl}(\cup \mathcal{N}) \subset \operatorname{cl} C_{\mathcal{N}} \backslash\left(\tilde{\partial} F_{\mathcal{N}} \cup(\cup \mathcal{N})\right)$.

e) It is clear that $C_{\mathcal{N}} \subset$ int $_{\overline{\mathbf{D}}} c l C_{\mathcal{N}}$. By Lemma 2, it suffices to show $\partial_{\overline{\mathbf{D}}} C_{\mathcal{N}} \subset$ $\partial_{\overline{\mathbf{D}}} c l C_{\mathcal{N}}$. Let $x \in \partial_{\overline{\mathbf{D}}} C_{\mathcal{N}}$. Then for any $\varepsilon>0$, there is $R \in \mathcal{N}$ such that $\left(B_{\varepsilon}(x) \cap \overline{\mathbf{D}}\right) \backslash$ cl $D_{\mathcal{N}}(R) \neq \emptyset$. (Indeed since $\partial_{\overline{\mathbf{D}}} C_{\mathcal{N}}=\left(\cup \mathcal{N}^{\circ}\right) \cup \operatorname{cl} \operatorname{ex} \mathcal{N}$ by d), we see that $x \in R^{\circ}$ for some $R \in \mathcal{N}$ or $x \in \operatorname{cl} \operatorname{ex} \mathcal{N}$. When $x \in \operatorname{cl} \operatorname{ex} \mathcal{N}$, there is $R \in \mathcal{N}$ such that $B_{\varepsilon}(x) \cap \operatorname{ex} R \neq \emptyset$.) So by (1), $\left(B_{\varepsilon}(x) \cap \overline{\mathbf{D}}\right) \backslash c l C_{\mathcal{N}}=\bigcup_{S \in \mathcal{N}}\left(B_{\varepsilon}(x) \cap \overline{\mathbf{D}}\right) \backslash c l D_{\mathcal{N}}(S) \neq \emptyset$. Thus $x \in c l C_{\mathcal{N}} \backslash \operatorname{int}_{\overline{\mathbf{D}}} c l C_{\mathcal{N}}=$ $\partial_{\overline{\mathbf{D}}} c l C_{\mathcal{N}}$.

f) By (1), $c l C_{\mathcal{N}}$ is closed and convex in C. Since $F_{\mathcal{N}} \in \mathcal{F}$, we have that $D=$ int $c l C_{\mathcal{N}}$ is convex and $c l D=c l C_{\mathcal{N}}$ by Theorems 6.2 and 6.3 in [3] (see also p. 44 in [3]). Therefore we see $D \subset$ int $_{\overline{\mathbf{D}}} c l C_{\mathcal{N}}=C_{\mathcal{N}} \subset \operatorname{cl} D$ by e). So $C_{\mathcal{N}}$ is connected. By (3), $C_{\mathcal{N}} \in \operatorname{Comp}(\overline{\mathbf{D}} \backslash \operatorname{cl}(\cup \mathcal{N}))$.

LEMMA 5. Let $\mathcal{L}$ be a lamination and $V \in \operatorname{Comp}(\overline{\mathbf{D}} \backslash \cup \mathcal{L})$ with $\sharp \mathcal{L}_{V} \geq 2$. Then

a) $F_{\mathcal{L}_{V}}=\partial \mathbf{D} \cap \mathrm{cl} V$. (Hence $\mathcal{L}_{V}=\mathcal{N}_{\partial \mathbf{D} \cap c l}$. $)$

b) For each $R \in \mathcal{L}_{+}$, there is $Q \in \mathcal{L}_{V}$ such that $D_{V}\langle Q\rangle \subset D_{V}\langle R\rangle$.

c) $C_{\mathcal{L}_{V}}=\operatorname{int}_{\overline{\mathbf{D}}}\left(\bigcap_{S \in \mathcal{L}_{+}} D_{V}\langle S\rangle\right)$.

d) $c l V=c l C_{\mathcal{L}_{V}}$.

PROOF. We prepare the following claim. Suppose $S \in \mathcal{S}_{+}$satisfies the condition that $\mathcal{L} \cup\{S\}$ is non-crossing and $S^{\circ} \cap V=\emptyset$. (Notice that $S \cap V=\emptyset$ because $V$ is relatively open in $\overline{\mathbf{D}}$ by Remark 1.) Then the following conditions are equivalent.

1) $S^{\circ} \cap \mathrm{cl} V \neq \emptyset$.

2) $S^{\circ} \subset D_{V}\langle R\rangle$ for any $R \in \mathcal{L}_{+} \backslash\{S\}$.

3) $S \in \mathcal{L}_{V}$.

4) $e x S \subset c l V$.

$\neg 2) \Rightarrow \neg 1)$ Since $\mathcal{L} \cup\{S\}$ is non-crossing, $S^{\circ} \cap$ cl $D_{V}\langle R\rangle=\emptyset$ for some $R \in \mathcal{L}_{+} \backslash\{S\}$. Then $S^{\circ} \cap c l V \subset S^{\circ} \cap c l D_{V}\langle R\rangle=\emptyset$. 2) $\Rightarrow 3$ ) Pick $x \in S^{\circ}$ and $y \in V$ (note $y \notin S$ and $x \neq y)$. Let $A$ be the half-open segment $(\operatorname{co}\{x, y\}) \backslash\{x\}$. So $D_{V}\langle S\rangle=D_{y}\langle S\rangle=D_{A}\langle S\rangle$. Let $R \in \mathcal{L}_{+} \backslash\{S\}$ (note $y \notin R$ ). Then $R \cap A=\emptyset$. (Indeed when $R^{\circ} \cap c l D_{V}\langle S\rangle=\emptyset$, it is clear. If $R^{\circ} \subset D_{V}\langle S\rangle$, then, by 2), $R \cap A=\emptyset$.) Therefore since $\mathcal{L} \cup\{S\}$ is non-crossing, $R \cap$ int $\Delta=\emptyset$ where $\Delta=\operatorname{co}(S \cup\{y\})$. Hence int $\Delta \subset \mathbf{D} \backslash \cup \mathcal{L}$. Furthermore since $y \in V \cap \Delta$ and int $\Delta$ is connected, we have int $\Delta \subset V$. Hence $S=\Delta \cap S \subset c l V \backslash V=\partial_{\overline{\mathbf{D}}} V$. 3) $\Rightarrow$ 1) is trivial. Thus 1), 2), 3) are equivalent. 3) $\Rightarrow 4$ ) is trivial. $\neg 2) \Rightarrow \neg 4)$ Since $S^{\circ} \cap c l D_{V}\langle R\rangle=\emptyset$ for some $R \in \mathcal{L}_{+} \backslash\{S\}$, we see ex $S \backslash$ cl $V \supset$ ex $S \backslash$ cl $D_{V}\langle R\rangle \neq \emptyset$.

Now we prove the lemma. Recall that $V \subset C_{\mathcal{L}_{V}}$ and $D_{\mathcal{L}_{V}}(R)=D_{V}\langle R\rangle$ for any $R \in \mathcal{L}_{V}$.

a) We show $\operatorname{Comp}\left(\partial \mathbf{D} \backslash F_{\mathcal{L}_{V}}\right)=\operatorname{Comp}(\partial \mathbf{D} \backslash \operatorname{cl} V)$ (hence $\left.F_{\mathcal{L}_{V}}=\partial \mathbf{D} \cap c l V\right)$.

Let $\gamma \in \operatorname{Comp}\left(\partial \mathbf{D} \backslash F_{\mathcal{L}_{V}}\right)$. By the definition of $F_{\mathcal{L}_{V}}, \gamma=\partial \mathbf{D} \backslash c l D_{\mathcal{L}_{V}}(R)=\partial \mathbf{D} \backslash$ cl $D_{V}\langle R\rangle$ for some $R \in \mathcal{L}_{V}$. Since $\tilde{\partial} \gamma=\operatorname{ex} R \subset c l V$, we have $\gamma \in \operatorname{Comp}(\partial \mathbf{D} \backslash c l V)$. Conversely let $\gamma \in \operatorname{Comp}(\partial \mathbf{D} \backslash c l V)$ and $\tilde{\partial} \gamma=\left\{a_{0}, a_{1}\right\}$. We show $\gamma=\partial \mathbf{D} \backslash c l D_{\mathcal{L}_{V}}(R)$ for some $R \in \mathcal{L}_{V}$ i.e. $S=\overline{a_{0} a_{1}} \in \mathcal{L}_{V}$. Since ex $S \subset \operatorname{cl} V$, it suffices to show $S$ satisfies the assumption in the above claim, that is, $\mathcal{L} \cup\{S\}$ is non-crossing and $S^{\circ} \cap V=\emptyset$. If there is $R \in \mathcal{L}_{+}$with $R^{\circ} \cap S \neq \emptyset$, then $a_{i} \notin c l V$ for some $i \in\{0,1\}$, contradicting the fact that 
$a_{i} \in \operatorname{cl} V$. So the family $\mathcal{L} \cup\{S\}$ is non-crossing. Assume there is $x \in S^{\circ} \cap V$. Pick $y \in \gamma$. Since $y \in \partial \mathbf{D} \backslash c l V$, there is $z \in \mathbf{D} \cap \operatorname{co}\{x, y\}$ with $z \in \partial_{\overline{\mathbf{D}}} V$. By Remark $1, z \in R^{\circ}$ for some $R \in \mathcal{L}_{V}$. Since $\mathcal{L} \cup\{S\}$ is non-crossing, $R^{\circ} \subset \operatorname{co} \gamma$. Hence $\gamma \cap$ ex $R \neq \emptyset$, contradicting the fact that $\gamma \cap$ ex $R \subset \gamma \cap \operatorname{cl} V=\emptyset$. So $S^{\circ} \cap V=\emptyset$.

b) Let $R \in \mathcal{L}_{+}$. Since $\partial \mathbf{D} \backslash$ cl $D_{V}\langle R\rangle \subset \partial \mathbf{D} \backslash c l V=\partial \mathbf{D} \backslash F_{\mathcal{L}_{V}}$, there is $\gamma \in \operatorname{Comp}(\partial \mathbf{D} \backslash$ $F_{\mathcal{L}_{V}}$ ) such that $\partial \mathbf{D} \backslash \operatorname{cl} D_{V}\langle R\rangle \subset \gamma$. Letting $Q=\operatorname{co} \tilde{\partial} \gamma$, we have $Q \in \mathcal{N}_{F_{\mathcal{L}_{V}}}=\mathcal{L}_{V}$ and $D_{V}\langle Q\rangle \subset D_{V}\langle R\rangle$.

c) By b), $\bigcap_{S \in \mathcal{L}_{V}} D_{\mathcal{L}_{V}}(S)=\bigcap_{S \in \mathcal{L}_{V}} D_{V}\langle S\rangle=\bigcap_{S \in \mathcal{L}_{+}} D_{V}\langle S\rangle$. Hence $C_{\mathcal{L}_{V}}=$ $\operatorname{int}_{\overline{\mathbf{D}}}\left(\bigcap_{S \in \mathcal{L}_{+}} D_{V}\langle S\rangle\right)$.

d) By Lemma 4-a), $c l C_{\mathcal{L}_{V}}=\left(\mathbf{D} \cap \operatorname{cl} C_{\mathcal{L}_{V}}\right) \cup F_{\mathcal{L}_{V}}$. Since $\mathbf{D}$ is open in $\mathbf{C}$, we see $\mathbf{D} \cap \operatorname{cl} C_{\mathcal{L}_{V}} \subset \operatorname{cl}\left(\mathbf{D} \cap C_{\mathcal{L}_{V}}\right)$. Hence $\operatorname{cl} C_{\mathcal{L}_{V}}=\operatorname{cl}\left(\mathbf{D} \cap C_{\mathcal{L}_{V}}\right) \cup F_{\mathcal{L}_{V}}$. By a), we can show that $c l V=\operatorname{cl}(\mathbf{D} \cap V) \cup F_{\mathcal{L}_{V}}$ in the same way as above. Therefore since $V \subset C_{\mathcal{L}_{V}}$, it suffices to show that $\mathbf{D} \cap C_{\mathcal{L}_{V}} \subset \mathbf{D} \cap V$. Let $x \in \mathbf{D} \cap C_{\mathcal{L}_{V}}$. By c), $B_{\varepsilon_{0}}(x) \subset \mathbf{D} \cap\left(\bigcap_{S \in \mathcal{L}_{+}} D_{V}\langle S\rangle\right)$ for some $\varepsilon_{0}>0$. In particular $B_{\varepsilon_{0}}(x) \subset \mathbf{D} \backslash \cup \mathcal{L}$. Since $B_{\varepsilon_{0}}(x)$ is connected, $B_{\varepsilon_{0}}(x) \subset V$.

COROLlaRY 1. Suppose that a lamination $\mathcal{L}$ satisfies the condition $\partial \mathbf{D}=c l$ ex $\mathcal{L}$. Then for any $V \in \operatorname{Comp}(\overline{\mathbf{D}} \backslash \cup \mathcal{L})$,

1) $V$ is open in $\mathbf{C}$ and $\sharp \mathcal{L}_{V} \geq 2$. (Hence $\mathcal{L}_{V}=\mathcal{N}_{\partial \mathbf{D} \cap \text { cl } V \text {.) }}$

2) $\widetilde{\operatorname{int}}(\partial \mathbf{D} \cap c l V)=\emptyset$.

3) $V=C_{\mathcal{L}_{V}}$.

Proof. 1) Since $\cup \mathcal{L}$ is closed in $\mathbf{C}$ and $\partial \mathbf{D}=\operatorname{cl} \operatorname{ex} \mathcal{L}$, we have $\partial \mathbf{D} \subset \cup \mathcal{L}$. Hence $V \subset \mathbf{D}$ and furthermore $V$ is open in $\mathbf{C}$. (Indeed since $V$ is relatively open in $\overline{\mathbf{D}}$ and $V \subset \mathbf{D}$, we see that for each $x \in V$, there is $\varepsilon>0$ with $B_{\varepsilon}(x) \subset V$.) We show $\sharp \mathcal{L}_{V} \geq 2$. Assume that $\mathcal{L}_{V}=\{S\}$ (recall Remark 1). Then $\mathbf{D} \cap D_{V}\langle S\rangle \subset V$. (Indeed assume there is $y \in$ $\mathbf{D} \cap D_{V}\langle S\rangle \backslash V$. Let $x \in V$. Then there is $z \in \operatorname{co}\{x, y\}$ such that $z \in \partial_{\overline{\mathbf{D}}} V$. Notice that $\operatorname{co}\{x, y\} \subset \mathbf{D} \cap D_{V}\langle S\rangle$. Since $z \in \mathbf{D} \cap \partial_{\overline{\mathbf{D}}} V$, there is $R \in \mathcal{L}_{V}$ such that $z \in R^{\circ}$ by Remark 1. Since $z \in D_{V}\langle S\rangle, R \neq S$, contradicting the assumption that $\mathcal{L}_{V}=\{S\}$.) There is $T \in \mathcal{L}_{+}$ with exT $\cap \partial \mathbf{D} \cap D_{V}\langle S\rangle \neq \emptyset$ because $\partial \mathbf{D}=\operatorname{cl} \operatorname{ex} \mathcal{L}$ and $\partial \mathbf{D} \cap D_{V}\langle S\rangle$ is relatively open in $\partial \mathbf{D}$. Since $\mathcal{L}$ is non-crossing, $T^{\circ} \subset \mathbf{D} \cap D_{V}\langle S\rangle \subset V$, contradicting the fact that $T \cap V=\emptyset$. By Lemma 5-a), $F_{\mathcal{L}_{V}}=\partial \mathbf{D} \cap c l V$ and $\mathcal{L}_{V}=\mathcal{N}_{\partial \mathbf{D} \cap c l V}$.

2) By 1), Lemma 4-b) and Lemma 5-c), we see that $\widetilde{\text { int }}(\partial \mathbf{D} \cap c l V)=\widetilde{\text { int }} F_{\mathcal{L}_{V}} \subset$ $C_{\mathcal{L}_{V}} \subset \bigcap_{S \in \mathcal{L}_{+}} D_{V}\langle S\rangle$. Assume $\widetilde{\operatorname{int}}(\partial \mathbf{D} \cap c l V) \neq \emptyset$. Since $\partial \mathbf{D}=c l$ ex $\mathcal{L}$, there is $S \in \mathcal{L}_{+}$

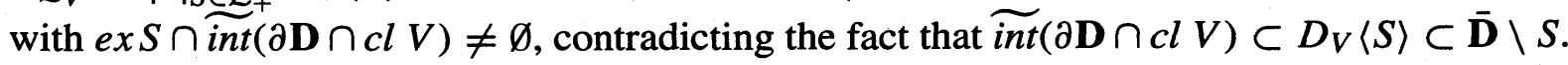

3) It suffices to show $C_{\mathcal{L}_{V}} \subset V$. By Lemma 5-c), $C_{\mathcal{L}_{V}} \cap\left(\cup \mathcal{L}_{+}\right)=\emptyset$. By 2) and Lemma 4-b), $C_{\mathcal{L}_{V}} \subset \mathbf{D}$. Hence $C_{\mathcal{L}_{V}} \subset \overline{\mathbf{D}} \backslash \cup \mathcal{L}$. Since $C_{\mathcal{L}_{V}}$ is connected (by Lemma 4-f)), $C_{\mathcal{L}_{V}} \subset V$.

\section{Gap invariance theorem.}

Let $d \in \mathbf{N}$ be $d \geq 2$. Define the mapping $p_{d}: \partial \mathbf{D} \rightarrow \partial \mathbf{D}$ by $p_{d}(z)=z^{d}$, and its iterated mappings, $p_{d}^{n}=p_{d}^{n-1} \circ p_{d}$ for each $n \in \mathbf{N}$ where $p_{d}^{0}$ is the identity mapping on 
$\partial \mathbf{D}$. For $z \in \partial \mathbf{D}$ and $n \geq 0$, define $p_{d}^{-n}(z)=\left\{w \in \partial \mathbf{D} \mid p_{d}^{n}(w)=z\right\}$. Note that for any $z \in \partial \mathbf{D}, \bigcup_{n \geq 0} p_{d}^{-n}(z)$ is dense in $\partial \mathbf{D}$. For each $S \in \mathcal{S}_{+}$, denote $P_{d} S=$ co $p_{d}($ ex $S)$. Let $\Omega_{d}=\left\{\exp \left(2 \pi i \frac{k}{d}\right) \mid k=0,1, \cdots, d-1\right\}$. For $S \in \mathcal{S}_{+}$and $\omega \in \Omega_{d}$, let $\omega S=\{\omega z \mid z \in S\} \in$ $\mathcal{S}_{+}$.

DEFINITION 4. Let $\mathcal{L}$ be a lamination. We say that $\mathcal{L}$ is a symmetric and invariant lamination under $p_{d}$ (we say d-SIL briefly) if the following three conditions hold:

(Symmetry) For any $S \in \mathcal{L}_{+}$and $\omega \in \Omega_{d}, \omega S \in \mathcal{L}$.

(Forward invariance) For any $S \in \mathcal{L}_{+}, P_{d} S \in \mathcal{L}_{+}$or $P_{d} S$ is degenerate.

(Backward invariance) For any $S \in \mathcal{L}_{+}$, there is $R \in \mathcal{L}$ such that $P_{d} R=S$.

Let $S \in \mathcal{S}$. Then $S=c o\{\exp (2 \pi i \alpha), \exp (2 \pi i \beta)\}$ for some $\alpha, \beta \in[0,1)$. Define $l(S)=\min \{|\alpha-\beta|, 1-|\alpha-\beta|\}$ and we call $l(S)$ the length of $S$. Then $0 \leq l(S) \leq 1 / 2$. Clearly $l(S)=0$ if and only if $S$ is degenerate, and $l(S)=1 / 2$ if and only if $S$ is a diameter.

REMARK 3. Let $\mathcal{L}$ be a d-SIL. Then $\partial \mathbf{D}=$ cl ex $\mathcal{L}$ and for each $S \in \mathcal{L}_{+}, l(S) \leq 1 / d$.

PROOF. Firstly we claim that for any $n \geq 0, p_{d}^{-n}$ ex $\mathcal{L} \subset$ ex $\mathcal{L}$. It suffices to show $p_{d}^{-1} \operatorname{ex} \mathcal{L} \subset$ ex $\mathcal{L}$. Let $z \in \operatorname{ex} S$ where $S \in \mathcal{L}_{+}$. By the backward invariance of $\mathcal{L}, P_{d} R=S$ for some $R \in \mathcal{L}$, in particular $y \in e x R$ for some $y \in p_{d}^{-1}(z)$. By the symmetry of $\mathcal{L}$, $p_{d}^{-1}(z)=\left\{\omega y \mid \omega \in \Omega_{d}\right\} \subset \bigcup_{\omega \in \Omega_{d}} \operatorname{ex}(\omega R) \subset \operatorname{ex} \mathcal{L}$. We show $\partial \mathbf{D}=$ clex $\mathcal{L}$. Let $z \in$ ex $\mathcal{L}$. Since $\bigcup_{n \geq 0} p_{d}^{-n}(z)$ is dense in $\partial \mathbf{D}$, we have for any $x \in \partial \mathbf{D}$ and $\varepsilon>0$, there is $y \in \bigcup_{n \geq 0} p_{d}^{-n}(z) \cap B_{\varepsilon}(x)$. By the above claim, $y \in \operatorname{ex} \mathcal{L}$. Next let $S \in \mathcal{L}_{+}$. By the noncrossing property and symmetry of $\mathcal{L}$, we see that the family $\left\{\omega S \mid \omega \in \Omega_{d}\right\}$ is non-crossing. Then $\sum_{\omega \in \Omega_{d}} l(\omega S) \leq 1$. Since $l(\omega S)=l(S), l(S) \leq 1 / d$.

Let $\mathcal{L}$ be a d-SIL. Then for each $V \in \operatorname{Comp}(\overline{\mathbf{D}} \backslash \cup \mathcal{L}), \mathcal{L}_{V}=\mathcal{N}_{\partial \mathbf{D} \cap c l} V$ by Remark 3 and Corollary 1-1). This is the key relation to prove gap invariance theorem. Define $P_{d}(c l V)=$ co $p_{d}(\partial \mathrm{D} \cap \mathrm{cl} V)$. Observe that

1) If $\sharp p_{d}(\partial \mathrm{D} \cap c l V)=1$, then $P_{d}(c l V)$ is a degenerate chord, that is, $P_{d}(c l V) \in$ $\mathcal{S} \backslash S_{+}$.

2) If $\sharp p_{d}(\partial \mathrm{D} \cap c l V)=2$, then $P_{d}(c l V) \in \mathcal{L}_{+}$.

Indeed let $p_{d}(\partial \mathbf{D} \cap c l V)=\left\{w_{1}, w_{2}\right\}$ where $w_{1} \neq w_{2}$. Since $\sharp(\partial \mathbf{D} \cap c l V)<\infty$ and $\mathcal{L}_{V}=\mathcal{N}_{\partial \mathbf{D} \cap c l}$, we see that each chord in $\mathcal{L}_{V}$ has to join two consecutive points in $\partial \mathbf{D} \cap c l V$ (recall the definition of $\left.\mathcal{N}_{\partial \mathbf{D} \cap c l V}\right)$. Hence there is $S \in \mathcal{L}_{V}$ such that $\operatorname{ex} S \cap p_{d}^{-1}\left(w_{k}\right) \neq \emptyset$ for each $k=1,2$. So $P_{d}(c l V)=c o\left\{w_{1}, w_{2}\right\}=\operatorname{co} p_{d}(\operatorname{ex} S)=P_{d} S \in \mathcal{L}$ by the forward invariance of $\mathcal{L}$.

In order to show gap invariance theorem, we use the following technical lemmas and will show them later. Let $\mathbf{0}$ be the origin of $\mathbf{C}$. When $S \in \mathcal{S}_{+}$is not a diameter (so $\mathbf{0} \notin S$ ), let $\gamma_{S}$ the open arc subtended by $S$, that is, $\gamma_{S}=\partial \mathbf{D} \backslash \mathrm{cl} D_{0}\langle S\rangle$.

LEMMA 6. Let $\mathcal{L}$ be ad-SIL. Then $\widetilde{\text { int }} p_{d}(\partial \mathbf{D} \cap c l V)=\emptyset$ for each $V \in \operatorname{Comp}(\overline{\mathbf{D}} \backslash \cup \mathcal{L})$.

LEMMA 7. Let $\mathcal{L}$ be a $d-S I L$ and $V \in \operatorname{Comp}(\overline{\mathbf{D}} \backslash \cup \mathcal{L})$. 
Suppose that $R \in \mathcal{S}_{+}$satisfies the conditions: $l(R)<1 / d, p_{d}(\operatorname{ex} R) \subset p_{d}(\partial \mathbf{D} \cap c l V)$ and $\bigcup_{\omega \in \Omega_{d}} \gamma_{\omega R} \subset \partial \mathbf{D} \backslash c l V$. Then $P_{d} R \in P_{d}\left(\mathcal{L}_{V}\right)_{+}$.

Gap InVARIANCE TheOREM. Let $\mathcal{L}$ be $d$-SIL and $V \in \operatorname{Comp}(\overline{\mathbf{D}} \backslash \cup \mathcal{L})$. Then $P_{d}(c l V) \in \mathcal{S} \backslash S_{+}$or $P_{d}(c l V) \in \mathcal{L}$, otherwise $P_{d}(c l V)$ is a gap of $\mathcal{L}:$ more precisely, int $P_{d}(c l V) \in \operatorname{Comp}(\overline{\mathbf{D}} \backslash \cup \mathcal{L})$ and $P_{d}(c l V)=$ cl int $P_{d}(c l V)$.

Proof. It suffices to show that if $\sharp p_{d}(\partial \mathbf{D} \cap c l V) \geq 3$, then int $P_{d}(c l V) \in \operatorname{Comp}(\overline{\mathbf{D}} \backslash$ $\cup \mathcal{L})$ and $P_{d}(c l V)=c l$ int $P_{d}(c l V)$. Suppose that $\sharp p_{d}(\partial \mathbf{D} \cap c l V) \geq 3$. By Lemma 6, $p_{d}(\partial \mathbf{D} \cap c l V)$ is disconnected. Hence we can define the 1 -sided family $\mathcal{N}=\mathcal{N}_{p_{d}(\partial \mathbf{D} \cap c l V)}$. Notice that $F_{\mathcal{N}}=p_{d}(\partial \mathbf{D} \cap c l V)$. Since $P_{d}(c l V)=c o F_{\mathcal{N}}$, we have by Lemma 4-c) and e),

$$
\begin{aligned}
& P_{d}(c l V)=c l C_{\mathcal{N}}, \\
& i n t_{\overline{\mathbf{D}}} P_{d}(c l V)=C_{\mathcal{N}}, \\
& \partial_{\overline{\mathbf{D}}} P_{d}(c l V)=\partial_{\overline{\mathbf{D}}} C_{\mathcal{N}} .
\end{aligned}
$$

At first we show that

$$
\mathcal{N}=P_{d}\left(\mathcal{L}_{V}\right)_{+}
$$

Let $S \in \mathcal{N}$. By the definition of $\mathcal{N}, S=\operatorname{co} \tilde{\partial} \gamma$ for some $\gamma \in \operatorname{Comp}\left(\partial \mathbf{D} \backslash F_{\mathcal{N}}\right)$. Denote $R=\operatorname{co} \tilde{\partial} \delta$ where $\delta \in \operatorname{Comp}\left(p_{d}^{-1}(\gamma)\right)$. Then $p_{d}(e x R)=\tilde{\partial} \gamma \subset F_{\mathcal{N}}(\operatorname{so} l(R)<1 / d)$. By the property of the mapping $p_{d}, \bigcup_{\omega \in \Omega_{d}} \gamma_{\omega R}=p_{d}^{-1}(\gamma) \subset p_{d}^{-1}\left(\partial \mathbf{D} \backslash F_{\mathcal{N}}\right) \subset \partial \mathbf{D} \backslash c l V$. By Lemma 7, $S=$ co $\tilde{\partial} \gamma=P_{d}(R) \in P_{d}\left(\mathcal{L}_{V}\right)_{+}$. Conversely let $R \in \mathcal{L}_{V}$ satisfy that $P_{d} R \in \mathcal{S}_{+}$. We show $P_{d}(R) \in \mathcal{N}$. By Remark $3, l(R)<1 / d$. Hence we see that $\omega_{1} R \cap \omega_{2} R=\emptyset$ if $\omega_{1}, \omega_{2} \in \Omega_{d}$ with $\omega_{1} \neq \omega_{2}$. By the symmetry of $\mathcal{L}, \omega R \in \mathcal{L}_{+}$for any $\omega \in \Omega_{d}$. Hence $V \subset C_{R}:=\left(\bigcap_{\omega \in \Omega_{d}} D_{0}\langle\omega R\rangle\right)$ or $V \subset \overline{\mathbf{D}} \backslash c l D_{0}\left\langle\omega_{0} R\right\rangle$ for some $\omega_{0} \in \Omega_{d}$. We have that $\partial \mathbf{D} \cap$ cl $V \subset \partial \mathbf{D} \cap \operatorname{cl} C_{R}$ or $\partial \mathbf{D} \cap c l V \subset \operatorname{cl} \gamma_{\omega_{0} R}$ for some $\omega_{0} \in \Omega_{d}$. Here notice that $p_{d}\left(c l \gamma_{\omega R}\right)=$ $p_{d}\left(c l \gamma_{R}\right)=c l p_{d}\left(\gamma_{R}\right)$ for each $\omega \in \Omega_{d}$ and $p_{d}\left(\partial \mathbf{D} \cap c l C_{R}\right) \cap p_{d}\left(\bigcup_{\omega \in \Omega_{d}} \gamma_{\omega R}\right)=\emptyset$ by the property of the mapping $p_{d}$. So $F_{\mathcal{N}} \cap p_{d}\left(\gamma_{R}\right)=\emptyset$ or $F_{\mathcal{N}} \subset c l p_{d}\left(\gamma_{R}\right)$. Since $\tilde{\partial} p_{d}\left(\gamma_{R}\right)=$ $p_{d}\left(\tilde{\partial} \gamma_{R}\right)=p_{d}(e x R) \subset F_{\mathcal{N}}$, we have that $p_{d}\left(\gamma_{R}\right) \in \operatorname{Comp}\left(\partial \mathbf{D} \backslash F_{\mathcal{N}}\right)$ or $\partial \mathbf{D} \backslash c l p_{d}\left(\gamma_{R}\right) \in$ $\operatorname{Comp}\left(\partial \mathbf{D} \backslash F_{\mathcal{N}}\right)$. By the definition of $\mathcal{N}, P_{d} R=\operatorname{co} \tilde{\partial} p_{d}\left(\gamma_{R}\right) \in \mathcal{N}$.

By the forward invariance of $\mathcal{L}$ and equality (4), we see that $\mathcal{N} \subset \mathcal{L}_{+}$.

Next we show the following equalities

$$
\begin{gathered}
\partial \mathbf{D} \cap \operatorname{cl} C_{\mathcal{N}} \cap(\cup \mathcal{L})=\tilde{\partial} F_{\mathcal{N}}, \\
\mathbf{D} \cap \operatorname{cl} C_{\mathcal{N}} \cap(\cup \mathcal{L})=\cup \mathcal{N}^{\circ} .
\end{gathered}
$$

By Remark 3, Lemma 4-a) and Lemma 6,

$$
\partial \mathbf{D} \cap c l C_{\mathcal{N}} \cap(\cup \mathcal{L})=\partial \mathbf{D} \cap c l C_{\mathcal{N}}=F_{\mathcal{N}}=\tilde{\partial} F_{\mathcal{N}}
$$

To prove (6), we give some preparation.

Define $\mathcal{B}=\left\{S \in \mathcal{L}_{+} \mid S^{\circ} \cap \operatorname{cl} C_{\mathcal{N}} \neq \emptyset\right\}$. Then

$$
\cup \mathcal{B} \subset \operatorname{cl} C_{\mathcal{N}} \quad \text { and } \quad \mathbf{D} \cap \operatorname{cl} C_{\mathcal{N}} \cap(\cup \mathcal{L})=\cup \mathcal{B}^{\circ} \text {. }
$$


(Indeed let $S \in \mathcal{B}$. Assume that $S^{\circ} \backslash c l C_{\mathcal{N}} \neq \emptyset$. Then $S^{\circ} \cap \partial_{\overline{\mathbf{D}}} c l C_{\mathcal{N}} \neq \emptyset$. By Lemma 4-d) and e), $S^{\circ} \cap\left(\cup \mathcal{N}^{\circ}\right) \neq \emptyset$, contradicting the fact that $\mathcal{N} \subset \mathcal{L}_{+}$and $\mathcal{L}$ is non-crossing. Hence we have $S \subset \operatorname{cl} C_{\mathcal{N}}$. Since $\mathbf{D} \cap(\cup \mathcal{L})=\cup \mathcal{L}^{\circ}$, the second statement holds.) Thus to prove (6), it suffices to show $\mathcal{N}=\mathcal{B}$. By Lemma 4-d), $\cup \mathcal{N}^{\circ} \subset \operatorname{cl} C_{\mathcal{N}}$. Since $\mathcal{N} \subset \mathcal{L}_{+}$, we have that $\mathcal{N} \subset \mathcal{B}$. Conversely let $S \in \mathcal{B}$. By the backward invariance of $\mathcal{L}, S=P_{d} R$ for some $R=\overline{x y} \in \mathcal{L}_{+}$. Then $l(R)<1 / d$ and $p_{d}(\operatorname{ex} R)=\operatorname{ex} S \subset \operatorname{exB} \subset \partial \mathbf{D} \cap \operatorname{cl} C_{\mathcal{N}}=F_{\mathcal{N}}=$ $p_{d}(\partial \mathbf{D} \cap c l V)$. Since $p_{d}^{-1}\left(p_{d}(x)\right)=\left\{\omega x \mid \omega \in \Omega_{d}\right\}$, we have that $\omega_{0} x \in c l V$ for some $\omega_{0} \in \Omega_{d}$. By the symmetry of $\mathcal{L}$, we can suppose $x \in c l V$. Furthermore by the symmetry of $\mathcal{L}$, we see that $V \subset C_{R}=\left(\bigcap_{\omega \in \Omega_{d}} D_{0}\langle\omega R\rangle\right)$ or $V \subset \overline{\mathbf{D}} \backslash \operatorname{cl} D_{0}\langle R\rangle$. Consider the case $c l V \subset \operatorname{cl} C_{R}$. Then $\bigcup_{\omega \in \Omega_{d}} \gamma_{\omega R} \subset \partial \mathbf{D} \backslash c l V$. So by Lemma 7 and the equality (4), we have that $S=P_{d} R \in P_{d}\left(\mathcal{L}_{V}\right)_{+}=\mathcal{N}$. Suppose that $c l V \subset \overline{\mathbf{D}} \backslash D_{0}\langle R\rangle$. Then $\partial \mathbf{D} \cap c l V \subset c l \gamma_{R}$. Furthermore since $p_{d}(y) \in p_{d}(\partial \mathbf{D} \cap c l V)$ and $l(R)<1 / d$, we see that $y$ has to belong to $c l V$. Then $\partial \mathbf{D} \backslash \operatorname{cl} \gamma_{R} \in \operatorname{Comp}(\partial \mathbf{D} \backslash \operatorname{cl} V)$. Hence $R \in \mathcal{N}_{\partial \mathbf{D} \cap c l V}=\mathcal{L}_{V}$. By the equality (4), $S=P_{d} R \in P_{d}\left(\mathcal{L}_{V}\right)_{+}=\mathcal{N}$.

By equalities (5), (6) and Lemma 4-d), we have

$$
c l C_{\mathcal{N}} \cap(\cup \mathcal{L})=\tilde{\partial} F_{\mathcal{N}} \cup\left(\cup \mathcal{N}^{\circ}\right)=\partial_{\overline{\mathbf{D}}} \mathcal{C}_{\mathcal{N}}
$$

Thus $C_{\mathcal{N}} \subset \overline{\mathbf{D}} \backslash \cup \mathcal{L}$. Note $\operatorname{cl}(\cup \mathcal{N}) \subset \cup \mathcal{L}$ since $\mathcal{N} \subset \mathcal{L}_{+}$and $\cup \mathcal{L}$ is closed. Hence $C_{\mathcal{N}} \in$ $\operatorname{Comp}(\overline{\mathbf{D}} \backslash \cup \mathcal{L})$ by Lemma 4-f). By Corollary 1-1), $C_{\mathcal{N}}$ is open in $\mathbf{C}$.

Therefore $C_{\mathcal{N}}=\operatorname{int} C_{\mathcal{N}}=\operatorname{int}\left(\operatorname{int}_{\overline{\mathrm{D}}} P_{d}(c l V)\right)=$ int $P_{d}(c l V)$, hence int $P_{d}(c l V) \in$ $\operatorname{Comp}(\overline{\mathbf{D}} \backslash \cup \mathcal{L})$ and furthermore we have $P_{d}(c l V)=c l C_{\mathcal{N}}=c l$ int $P_{d}(c l V)$.

(Proof of LemMa 6) Case 1. $\quad l(S)=1 / 2$ for some $S \in \mathcal{L}_{V}$.

By Remark 3, $d=2$. Notice that $p_{2}(\partial \mathbf{D} \cap c l V)=p_{2}(\partial \mathbf{D} \cap c l V \backslash e x S) \cup p_{2}($ ex $S)$, $\partial \mathbf{D} \cap c l V \backslash e x S \subset \partial \mathbf{D} \cap D_{V}\langle S\rangle$ and $p_{2}$ is injective on $\partial \mathbf{D} \cap D_{V}\langle S\rangle$. By Corollary 1-2), $\widetilde{\text { int }} p_{2}(\partial \mathbf{D} \cap c l V \backslash$ ex $S)=\emptyset$. Hence $\widetilde{\text { int }} p_{2}(\partial \mathbf{D} \cap c l V)=\emptyset$.

Case 2. $l(S)<1 / 2$ for some $S \in \mathcal{L}_{V}$.

Then $\mathrm{0} \notin \partial_{\overline{\mathrm{D}}} V$ by Remark 1 . Thus $\mathrm{0} \notin c l V$ or $\mathrm{0} \in V$.

Suppose $\mathbf{0} \notin c l V$. Let $x \in V$. Then there is $y \in \operatorname{co}\{\mathbf{0}, x\} \cap \partial_{\overline{\mathbf{D}}} V \backslash\{\mathbf{0}, x\}$. By Remark 1 , $y \in R$ for some $R \in \mathcal{L}_{V}$. Since $\mathbf{0} \notin c l V$, we see that $\partial \mathbf{D} \cap c l V \backslash$ ex $R \subset \gamma_{R}$. Since $l(R) \leq 1 / d$ (by Remark 3), $p_{d}$ is injective on $\gamma_{R}$. By Corollary 1-2), $\widetilde{\text { int }} p_{d}(\partial \mathbf{D} \cap c l V \backslash$ ex $R)=\emptyset$. Hence $\widetilde{\text { int }} p_{d}(\partial \mathbf{D} \cap \mathrm{cl} V)=\emptyset$.

Suppose $\mathbf{0} \in V$. Then $\omega R \in \mathcal{L}_{V}$ for any $R \in \mathcal{L}_{V}$ and $\omega \in \Omega_{d}$. (Indeed let $R \in \mathcal{L}_{V}$. Assume that $\omega R \notin \mathcal{L}_{V}$ for some $\omega \in \Omega_{d}$. By the symmetry of $\mathcal{L}, \omega R \in \mathcal{L}_{+}$. By Corollary 1-1) and Lemma 5-b), $(\omega R)^{\circ} \cap D_{0}\langle Q\rangle=\emptyset$ for some $Q \in \mathcal{L}_{V}$. By the symmetry of $\mathcal{L}$, $\omega^{-1} Q \in \mathcal{L}_{+}$. Furthermore $R^{\circ} \cap D_{0}\left\langle\omega^{-1} Q\right\rangle=\emptyset$, contradicting the fact that $R \in \mathcal{L}_{V}$.) So since $\mathcal{N}_{\partial \mathbf{D} \cap c l V}=\mathcal{L}_{V}$, we see that $\{\omega z \mid z \in \gamma\} \in \operatorname{Comp}(\partial \mathbf{D} \backslash c l V)$ for each $\gamma \in \operatorname{Comp}(\partial \mathbf{D} \backslash c l V)$ and $\omega \in \Omega_{d}$. Thus $\partial \mathbf{D} \cap c l V$ is invariant under the rotation of angle $2 \pi / d$. Let $z \in c l V$ and $z_{1}=z \exp (2 \pi i / d)$. So $p_{d}(\partial \mathbf{D} \cap c l V)=p_{d}\left(\partial \mathbf{D} \cap c l V \cap \gamma \overline{z z_{1}}\right) \cup\left\{p_{d}(z)\right\}$. Since $p_{d}$ is injective on $\gamma \overline{z z_{1}}$, int $p_{d}(\partial \mathbf{D} \cap c l V)=\emptyset$ by Corollary 1-2). 
(Proof OF LEMMA 7) Suppose that $R=\overline{y z} \in \mathcal{S}_{+}$satisfies the conditions: $l(R)<$ $1 / d, p_{d}($ ex $R) \subset p_{d}(\partial \mathbf{D} \cap c l V)$ and $\bigcup_{\omega \in \Omega_{d}} \gamma_{\omega R} \subset \partial \mathbf{D} \backslash c l V$. So $P_{d} R \in \mathcal{S}_{+}$. We shall show that $P_{d} R \in P_{d}\left(\mathcal{L}_{V}\right)$. Note that $p_{d}^{-1}\left(p_{d}(z)\right)=\left\{\omega z \mid \omega \in \Omega_{d}\right\}$. Hence $\omega_{0} z \in c l V$ for some $\omega_{0} \in \Omega_{d}$ because $p_{d}(z) \in p_{d}(\partial \mathbf{D} \cap c l V)$. Since $P_{d} R=P_{d}\left(\omega_{0} R\right)$, we can suppose $z \in c l V$ without loss of generality. Since $z \in c l V$ and $\gamma_{R} \subset \partial \mathbf{D} \backslash c l V$, we see that

there is $\gamma \in \operatorname{Comp}(\partial \mathbf{D} \backslash c l V)$ such that $\gamma_{R} \subset \gamma$ and $z \in \tilde{\partial} \gamma$.

Let $\tilde{\partial} \gamma=\{x, z\}$. Hence $S=\overline{x z} \in \mathcal{N}_{\partial \mathbf{D} \cap c l} V=\mathcal{L}_{V}$ and $x \in c l V$. Notice that

$$
\text { if } l(S)<\frac{1}{2}, \quad \text { then } \gamma=\gamma_{S} \text { or } \gamma=\partial \mathbf{D} \backslash c l \gamma_{S} \text {. }
$$

Let $y_{k}=y \exp (2 \pi i k / d)$ and $z_{k}=z \exp (2 \pi i k / d)$ where $k \in\{1, d-1\}$.

Case 1. $x=z_{k}$ where $k \in\{1, d-1\}$ (or equivalently, $l(S)=1 / d$ ).

Suppose $d=2$. Then $x=-z$. Since $l(R)<1 / 2, y \in \gamma$. In particular $y \notin c l V$. Since $p_{2}(y) \in p_{2}(\partial \mathbf{D} \cap c l V),-y$ has to belong to $c l V$. Since $\gamma_{-R} \cap c l V=\emptyset$ and $e x(-R) \subset c l V$, we have $-R \in \mathcal{N}_{\partial \mathrm{D} \cap c l} V=\mathcal{L}_{V}$. Hence $P_{2} R=P_{2}(-R) \in P_{2}\left(\mathcal{L}_{V}\right)$.

Suppose $d \geq 3$. Then $l(s)=1 / d<1 / 2$. Firstly we show $\gamma=\partial \mathbf{D} \backslash c l \gamma_{s}$.

Assume that $\gamma=\gamma_{S}$. Then $V \subset D_{0}\langle S\rangle$. Furthermore

$$
c l V=P \quad \text { where } P=\operatorname{co~} p_{d}^{-1}\left(p_{d}(z)\right) .
$$

(Indeed by the symmetry of $\mathcal{L}$, we see that $\omega S \in \mathcal{L}$ for any $\omega \in \Omega_{d}$. Notice that $\omega S$ is a side of the polygon $P$. If there is $x \in(\cup \mathcal{L}) \cap$ int $P$, then there is $R \in \mathcal{L}_{+}$such that $x \in R^{\circ}$, but $R^{\circ} \cap\left(\bigcup_{\omega \in \Omega_{d}} \omega S\right) \neq \emptyset$, contradicting the non-crossing property of $\mathcal{L}$. So int $P \subset \overline{\mathbf{D}} \backslash \cup \mathcal{L}$. Since int $P$ is connected and contained in $D_{0}\langle S\rangle$, we have that int $P=V$.) So since $p_{d}(y) \in$ $p_{d}(\partial \mathbf{D} \cap c l V)$, we see that $p_{d}^{-1}\left(p_{d}(y)\right) \cap p_{d}^{-1}\left(p_{d}(z)\right)=p_{d}^{-1}\left(p_{d}(y)\right) \cap \partial \mathbf{D} \cap c l V \neq \emptyset$. Hence $p_{d}(y)=p_{d}(z)$, contradicting the fact that $P_{d} R \in \mathcal{S}_{+}$.

Therefore $\partial \mathbf{D} \backslash c l \gamma_{S}=\gamma \subset \partial \mathbf{D} \backslash c l V$ i.e. $\partial \mathbf{D} \cap c l V \subset c l \gamma_{S}$. Since $\gamma_{R} \subset \partial \mathbf{D} \backslash c l \gamma_{S}$ and $l(R)<l(S)$, we see that $y \notin c l \gamma_{S}$. Since $S=\overline{z z_{k}}$, there is unique $\omega \in \Omega_{d}$ such that $\omega y \in c l \gamma_{s}$. Hence $\omega y=y_{k}$ i.e. $\omega=\exp (2 \pi i k / d)$. Furthermore $y_{k}$ has to belong to $c l V$ because $p_{d}(y) \in p_{d}(\partial \mathbf{D} \cap c l V) \subset p_{d}\left(c l \gamma_{S}\right)$. Hence ex $(\omega R)=\left\{z_{k}, y_{k}\right\}=\left\{x, y_{k}\right\} \subset c l V$. Since $\gamma_{\omega R} \cap c l V=\emptyset$, we have $\omega R \in \mathcal{N}_{\partial \mathrm{D} \cap c l V}=\mathcal{L}_{V}$. Hence $P_{d} R=P_{d}(\omega R) \in P_{d}\left(\mathcal{L}_{V}\right)$.

Case 2. $x \notin\left\{z_{1}, z_{d-1}\right\}$.

Then $l(S)<1 / d$ by Remark 3. There is unique $k \in\{1, d-1\}$ with $x \in \sqrt{\overline{z z k}}$, and there is unique $l \in\{1, d-1\}$ with $y \in \gamma \overline{z z l}$.

Suppose $k \neq l$. Then $x \in \gamma \overline{y_{k} z} \cup\left\{y_{k}\right\}$ because $\bigcup_{\omega \in \Omega_{d}} \gamma_{\omega R} \subset \partial \mathbf{D} \backslash c l V$ and $x \in c l V \backslash\{z\}$. So $\gamma_{R} \cap c l \gamma_{S}=\emptyset$, and if $x \neq y_{k}$, then $p_{d}^{-1}\left(p_{d}(y)\right) \cap c l \gamma_{S}=\emptyset$. Since $\gamma_{R} \subset \gamma, \gamma=\partial \mathbf{D} \backslash c l \gamma_{S}$, that is, $\partial \mathbf{D} \cap c l V \subset c l \gamma_{S}$. Since $p_{d}(y) \in p_{d}\left(c l \gamma_{S}\right), x=y_{k}$. So $P_{d} R=P_{d} S \in P_{d}\left(\mathcal{L}_{V}\right)$.

Suppose $k=l$. Assume that $R \neq S$. Then $y \in \gamma_{S}=\gamma$. (Indeed if $y \notin \gamma_{S}$, then since $x \neq y, x \in \gamma_{R} \cap c l V$, contradicting the assumption that $\gamma_{R} \cap c l V=\emptyset$. So $\gamma=\gamma_{S}$ since $y \in \gamma_{S}$ and $\gamma_{R} \subset \gamma$.) Therefore $\partial \mathbf{D} \cap c l V \subset \partial \mathbf{D} \backslash \gamma_{S}$ because $\gamma_{S}=\gamma$. Since $y \in \gamma_{S}$ and $p_{d}(y) \in p_{d}(\partial \mathbf{D} \cap c l V)$, we have that there is $\omega \in \Omega_{d}$ such that $\omega y \in c l V$ and $\omega \neq 1$. Since 
$\omega S \in \mathcal{L}_{+}$(by the symmetry of $\mathcal{L}$ ) and $\omega y \in \gamma_{\omega S} \cap c l V$, we have $\partial \mathbf{D} \cap c l V \subset c l \gamma_{\omega S}$. Since $\omega \neq 1$ and $l(\omega S)<1 / d$, we see $z \notin c l \gamma_{\omega S}$, contradicting the assumption that $z \in c l V$.

\section{Appendix.}

(Proof of Lemma 1) Since $\partial E \subset \partial D \subset X \backslash D, D \cap c l E=D \cap$ int $E \subset$ int $E \subset$ $E \subset D \cap c l E$ and hence int $E=E$ and furthermore $E=D \cap c l E$ i.e. $E$ is a non-empty and relative clopen subset of $D$. Then $E=D$ by the connectivity of $D$.

(Proof OF LEMMA 2) Notice that $\partial E=(c l E) \backslash($ int $E)$ and $\partial c l E=(c l E) \backslash$ (int cl E).

(Proof of Lemma 3) Since $C$ is relatively closed in $X \backslash F, C=(c l C) \cap(X \backslash F)$. Since $C$ is open in $X, \partial C=(\mathrm{cl} C) \backslash C=(\mathrm{cl} C) \cap F \subset F$.

For the second statement, it suffices to show that $\partial F \subset \operatorname{cl}\left(\bigcup_{C \in \operatorname{Comp}(X \backslash F)} \partial C\right)$. Let $x \in$ $\partial F$ and $U$ be an open subset of $X$ with $x \in U$. Denote by $D$ the component of $U$ containing $x$. Then $D \backslash F \neq \emptyset$ since $D$ is open in $X$ and $x \in \partial F$. Hence there is $C_{0} \in \operatorname{Comp}(X \backslash F)$ such that $D \cap C_{0} \neq \emptyset$. Here $x \in D \cap F \subset D \backslash C_{0}$. Thus $D \cap C_{0} \neq \emptyset \neq D \backslash C_{0}$. Since $D$ is connected, $U \cap\left(\bigcup_{C \in \operatorname{Comp}(X \backslash F)} \partial C\right) \supset D \cap \partial C_{0} \neq \emptyset$. Hence $x \in \operatorname{cl}\left(\bigcup_{C \in \operatorname{Comp}(X \backslash F)} \partial C\right)$.

\section{References}

[1] C. BANDT and K. KELler, Symbolic dynamics for angle-doubling on the circle, I, Ergodic Theory and Related Topics III (eds. U. KRENGEL, K. RICHTER and V. WARSTAT), Lecture Notes in Math. 1514 (1992), Springer, 1-23.

[ 2 ] K. M. Pilgrim, Rational maps whose Fatou components are Jordan domains, Ergod. Th. and Dynam. Sys. 16 (1996), 1323-1343.

[ 3 ] R. T. Rockafellar, Convex Analysis, Princeton Univ. Press.

[4] W. P. THURSTON, On the geometry and dynamics of iterated rational maps, preprint.

Present Address:

Department of MAThematics, Osaka City University, SUGIMOTO, SUMIYOSHI-KU, OSAKA, 558-8585 JAPAN.

e-mail: yoshida@sci.osaka-cu.ac.jp 